\title{
Simplified hydrodynamic analysis of superfluid turbulence in He II: Internal dynamics of inhomogeneous vortex tangle
}

\author{
J. A. Geurst \\ Department of Mathematics and Informatics, Delft University of Technology, P.O. Box 5031, 2600 GA Delft, The Netherlands \\ H. van Beelen \\ Kamerlingh Onnes Laboratory, Leiden University, P.O. Box 9506, 2300 RA Leiden, The Netherlands
}

(Received 30 January 1996; revised manuscript received 16 April 1996)

\begin{abstract}
The hydrodynamic theory of superfluid turbulence is presented in a new simplified form. It applies to flow situations frequently encountered in practice, in which the thermohydrodynamic environment of a superfluid turbulent tangle of quantized vortices may be considered, in a first order of approximation, as given. Flow quantities like the mass density, the entropy density, and the drift velocities of mass and elementary excitations act, accordingly, as external parameters with respect to the internal dynamics of the vortex tangle. The internal dynamics is completely specified by a kinematic equation governing the time evolution of the line-length density of the quantized vortices and a dynamic equation involving the impulse density of the vortex tangle. The derivation of these equations starts from a variational principle that is reminiscent of Hamilton's principle in classical mechanics and proceeds, in order to include dissipative effects, by using methods of the thermodynamics of irreversible processes. A new quantity called superfluid turbulent pressure is introduced which shows many properties that are familiar from the ordinary pressure in a classical fluid. Two important particular cases are considered in more detail, viz., homogeneous superfluid turbulent flow and flow situations in which the vortex tangle is in permanent internal equilibrium. When diffusion of the vortex-tangle impulse is taken into account and dispersive effects are disregarded, the dynamic equation of the vortex tangle assumes, in the case of internal equilibrium, the form of Burgers' equation with a nonlinear source term. This equation, which is new, may be considered as a natural generalization of Vinen's equation to inhomogeneous superfluid turbulence. Some exact solutions which represent uniformly propagating superfluid turbulence fronts are listed in the Appendix. [S0163-1829(96)01033-8]
\end{abstract}

\section{INTRODUCTION}

The general hydrodynamic three-fluid theory of superfluid turbulence in He II developed for one-dimensional flow in (Ref. 1) and for three-dimensional flow in (Ref. 2) is fairly involved. In fact, three scalar and three vector evolution equations are required for the determination, as a function of position and time, of three scalar quantities $\rho, S$, and $L$ representing, respectively, the local densities of mass, elementary excitations - or entropy — and line length of the quantized vortices constituting a vortex tangle, and three vector quantities $\vec{v}, \vec{v}_{n}$, and $\vec{v}_{\ell}$ denoting, respectively, the drift velocities of mass, elementary excitations, and quantized vortices. We are therefore interested in special cases where the three-fluid equations take a particularly simple form.

A good example of such a relatively simple form of the three-fluid theory is afforded by the homogeneous case in which the flow quantities do not vary with position. The case of homogeneous flow is considered in detail in Ref. 3. In addition, a section is devoted to it in Ref. 2 . The decay of homogeneous superfluid turbulence is analyzed in Refs. 4 and 5. For the analysis of homogeneous flow it appears to be sufficient to supplement the two-fluid equations for superfluid ${ }^{4} \mathrm{He}$ (Landau-Khalatnikov equations), irrespective of an additional mutual-friction force, with a slightly extended form of Vinen's equation governing the time evolution of the line-length density $L$ of the quantized vortices. The extension of Vinen's equation involves the appearance of the relative polarity of the vortex tangle, i.e., the cosine of the angle between the direction of the relative drift velocity $\vec{w}_{\ell}=\vec{v}_{\ell}-\vec{v}$ of the vortex tangle and the direction of the relative drift velocity $\vec{w}_{n}=\vec{v}_{n}-\vec{v}$ of the elementary excitations. In one-dimensional flow the relative polarity takes the form $\operatorname{sgn} w_{\ell} \operatorname{sgn} w_{n}$. It plays an important part in the analysis of the decay of superfluid turbulence (see, in particular, Ref. 5).

Although homogeneous superfluid turbulent flow appears to be understood reasonably well now, both experimentally ${ }^{6}$ and theoretically, ${ }^{7}$ inhomogeneous flow phenomena like superfluid turbulence fronts still await a simple unifying treatment. It is the aim of this paper to provide such a treatment by applying only a few basic principles. In particular we are looking for equations governing inhomogeneous superfluid turbulent flow that generalize Vinen's equation in a natural way.

In a recent paper $^{8}$ dealing with the propagation of superfluid turbulence fronts a class of flow situations is envisaged in which the thermohydrodynamic environment ("bath") in which the vortex tangle is embedded, is regarded as given, usually constant with respect to position and time, while the vortex tangle itself is allowed to evolve according to its own dynamics. The influence of the thermohydrodynamic environment on the dynamics of the vortex tangle is represented by the external parameters $\rho, S, \vec{v}$, and $\vec{v}_{n}$. The vortex linelength density $L$ and the relative drift velocity $\vec{w}_{\ell}$ of the 
quantized vortices constitute the internal variables characterizing the various superfluid turbulent states of the tangle.

We want to develop here, in an independent way, for the class of flow situations just mentioned, the appropriate simplified form of our hydrodynamic theory of superfluid turbulence. It should be noted that flow situations of this kind are, approximately, realized in a variety of experiments. Since it turns out to be sufficient, in many cases of practical interest, to consider one-dimensional flow only, the internal variables involved in the dynamics of a vortex tangle will be represented by the scalar quantities $L$ and $w_{\ell}$.

In Sec. II the nondissipative equation of motion for a superfluid turbulent tangle of quantized vortices is derived from an appropriate variational principle which is reminiscent of Hamilton's principle in classical mechanics. According to that derivation the impulse density $P_{\ell}$ of the vortex tangle is determined irrespective of an arbitrary function of the dimensionless Vinen number $V i=\kappa L^{1 / 2} /\left|w_{\ell}\right|$, where $\kappa=h / m$. The specification of this function follows from an analysis of the energy of the vortex tangle in Sec. III. A superfluid turbulent pressure $p_{\ell}$ satisfying the ClebschBateman principle is also introduced in that section. Dissipative effects like mutual-friction forces are added in Sec. IV according to the thermodynamics of irreversible processes. The diffusion of vortex-tangle impulse receives explicit attention. After a review of homogeneous flow in Sec. V the particular form taken by the equations when a condition of permanent internal equilibrium is imposed on the vortex tangle, is investigated in Sec. VI. When dispersive effects are neglected that particular form reduces to Burgers' equation with a nonlinear source term. Some exact solutions of this equation representing uniformly propagating superfluid turbulence fronts are listed in the Appendix. The basic equations of the paper are finally reviewed in Sec. VII.

Theoretical and experimental results on homogeneous superfluid turbulent flow are comprehensively reviewed by Tough. ${ }^{6}$ For an introduction to the subject we refer to the book by Donnelly, ${ }^{9}$ where a chapter is devoted to superfluid turbulence. An interesting review of recent results on inhomogeneous superfluid turbulence is presented by $\mathrm{Ne}$ mirovskii and Fiszdon. ${ }^{10}$

\section{VARIATIONAL PRINCIPLE FOR NONDISSIPATIVE SUPERFLUID TURBULENCE}

As set forth in the preceding section we like to develop, for flow situations in which the tangle of quantized vortices may approximately be taken to be immersed in a given environment of $\mathrm{He}$ II, the appropriate one-dimensional simplified version of our hydrodynamic theory of superfluid turbulent flow. The given environment is, in a first order of approximation, characterized by definite values of the external parameters $\rho, S, v$, and $v_{n}$ which, in general, will vary with position and time. Although the values of quantities like the temperature gradient may be affected as a result of the dynamics of the vortex tangle, they will do so only in a second order of approximation (see Appendix A of Ref. 8). The flow conditions just mentioned are encountered in a variety of experimental situations. For instance, in capillary flow the external parameters may be treated as constants provided the flow velocities are small compared to the propaga- tion velocities of first and second sound and, in addition, the boundary conditions at the entrance and exit of the capillary are time independent (cf. Ref. 8; see also the first paragraph of Sec. III).

The internal dynamics of the vortex tangle will be characterized by two time-dependent fields, viz., $L(x, t)$ representing the local length of quantized vortices per unit volume, and $w_{\ell}(x, t)$ denoting the relative drift velocity $v \ell-v$ of the vortex tangle. It may be demonstrated by starting from the "microscopic" equation of motion for a quantized line vortex (see the Appendix in Ref. 3) that, when dissipative effects are disregarded, the line length of the vortices is conserved, i.e.,

$$
\frac{\partial L}{\partial t}+\frac{\partial}{\partial x}\left(L v_{\ell}\right)=0
$$

We shall refer to Eq. (1) as the kinematic equation of a vortex tangle in the nondissipative case. The equation of motion being valid in that case will be derived from a variational principle, viz.,

$$
\delta \int_{t_{0}}^{t_{1}} d t \int_{x_{0}}^{x_{1}} \Lambda / d x=0
$$

where the Lagrangian density $\Lambda_{\ell}$ is a function of the external parameters $\rho, S, w_{n}$ and the internal variables $L$, $\partial L / \partial x$, and $w_{\ell}$, i.e.,

$$
\Lambda_{\ell}=\Lambda_{\ell}\left(\rho, S, w_{n} ; L, \partial L / \partial x, w_{\ell}\right) .
$$

Note that

$$
w_{n}=v_{n}-v, \quad w_{\ell}=v_{\ell}-v .
$$

The variation of the internal variables in Eq. (2) is subject to the kinematic constraint (1). The Lagrangian density will be written in the following more specific form which is reminiscent of Hamilton's principle in classical mechanics:

$$
\Lambda_{\ell}=\frac{1}{2} \tilde{m}_{\ell} w_{\ell}^{2}-\widetilde{U}_{\ell} .
$$

The expression at the right-hand side of Eq. (5) is, however, quite general. In fact, $\tilde{m}_{\ell}$ having the dimension of mass density is an as yet unknown function of the external parameters and the internal variables $L$ and $w_{\ell}$ which may, formally, be expressed by

$$
\tilde{m}_{\ell}=\tilde{m}_{\ell}\left(\rho, S, w_{n} ; L, w_{\ell}\right) .
$$

The potential density $\widetilde{U}_{\ell}$, however, has a definite meaning; it represents the internal energy density of the vortex tangle according to

$$
\widetilde{U}_{\ell}=\rho_{s} \frac{\kappa^{2}}{4 \pi} L \ln \left(\frac{c}{a_{0} L^{1 / 2}}\right)+\frac{1}{2} \widetilde{g} \ell\left(\frac{\partial L}{\partial x}\right)^{2},
$$

where the mass density of the superfluid component is indicated by $\rho_{s}$, the quantum of circulation $\kappa$ equals $h / m$ and $a_{0} \approx 1.3 \AA$ (see Ref. 9) denotes the core radius of a quantized line vortex. The first term on the right-hand side of Eq. (7) represents the density of the "microscopic" kinetic energy associated with the circulating motion around the core of the quantized vortices [see Khalatnikov (Ref. 11)]. This expres- 
sion is, approximately, valid provided $a_{0} L^{1 / 2} \ll 1$. The dimensionless constant $c$ is of order unity. The gradient $\partial L / \partial x$ appears only in the second term at the right-hand side of Eq. (7). This term, in fact, models, within the framework of the macroscopic theory, the effects associated with local deviations from macroscopic behavior caused by very large values of the gradient $\partial L / \partial x$. Large gradients may appear, e.g., in sharp boundary layers and steep fronts. The coefficient $\widetilde{g}_{\ell}$ is assumed to depend on the external parameters and the single internal variable $L$. A dimensional analysis then shows that

$$
\tilde{g_{\ell}}=\rho_{s} \frac{\kappa^{2}}{L^{2}} \gamma_{\ell},
$$

where $\gamma_{\ell}$ is, in general, a dimensionless function of the external parameters, i.e.,

$$
\gamma_{\ell}=\gamma_{\ell}\left(\rho, S, w_{n}\right) .
$$

In practice, however, $\gamma_{\ell}$ will, effectively, be a function of the absolute temperature, e.g., through $\rho_{s} / \rho$. Since the coefficient $\tilde{m}_{\ell}$ may depend on the internal variables $L$ and $w_{\ell}$ a dimensional analysis shows that

$$
\tilde{m}_{\ell}=\rho_{s} \hat{m}_{\ell},
$$

where the dimensionless coefficient $\hat{m}_{/}$depends, irrespective of the external parameters, exclusively on the following dimensionless combination $\mathrm{Vi}$ (Vinen number) of the internal variables:

$$
V i=\kappa L^{1 / 2} /\left|w_{\ell}\right| .
$$

Possible cross effects between internal and external variables are accordingly suppressed, in view of our intention to model the internal dynamics of a vortex tangle. The functional dependence of $\hat{m}_{\ell}$ is, in a qualitative way, expressed by

$$
\hat{m}_{\ell}=\hat{m}_{\ell}\left(\rho, S, w_{n} ; V i\right) .
$$

Note that the dependence on the Vinen number $V i$ is as yet unspecified (see, however, the next section).

After putting

$$
\Lambda_{\ell}=-U_{\ell},
$$

where $U_{\ell}$ may be considered as the extended internal energy density of the vortex tangle, we have in view of Eq. (5)

$$
U_{\ell}=\widetilde{U}_{\ell}-\frac{1}{2} \tilde{m}_{\ell} w_{\ell}^{2},
$$

so that

$$
d U_{\ell}=\mu / d L+g_{\ell} d\left(\frac{\partial L}{\partial x}\right)-\frac{1}{2} m / d\left(w_{\ell}^{2}\right) .
$$

In this expression $\mu_{\ell}$ represents the line-length potential determined by

$$
\mu_{\ell}=\rho \kappa \beta_{v}+\frac{1}{2} \frac{\partial \tilde{g}_{\ell}}{\partial L}\left(\frac{\partial L}{\partial x}\right)^{2}-\frac{1}{2} \frac{\partial \tilde{m}_{\ell}}{\partial L} w_{\ell}^{2},
$$

where

$$
\beta_{v}=\frac{\rho_{s}}{\rho} \frac{\kappa}{4 \pi} \ln \left(\frac{c^{\prime}}{a_{0} L^{1 / 2}}\right)
$$

with

$$
\ln c^{\prime}=\ln c-\frac{1}{2},
$$

while the quantities $g_{\ell}$ and $m_{\ell}$ are given by

$$
g_{\ell}=\tilde{g} \ell \frac{\partial L}{\partial x}
$$

and

$$
m_{\ell}=\tilde{m}_{\ell}+\frac{\partial \tilde{m}_{\ell}}{\partial\left(w_{\ell}^{2}\right)} w_{\ell}^{2} .
$$

We have according to Eqs. (8), (10), and (12)

$$
\begin{gathered}
\frac{1}{2} \frac{\partial \tilde{g}_{\ell}}{\partial L}\left(\frac{\partial L}{\partial x}\right)^{2}=-\rho_{s} \frac{\kappa^{2}}{L^{3}} \gamma_{\ell}\left(\frac{\partial L}{\partial x}\right)^{2}, \\
-\frac{1}{2} \frac{\partial \tilde{m}_{\ell}}{\partial L} w_{\ell}^{2}=-\frac{1}{4} \kappa^{2} \frac{1}{V i} \frac{\partial \tilde{m}_{\ell}}{\partial(V i)}, \\
\frac{\partial \tilde{m}_{\ell}}{\partial\left(w_{\ell}^{2}\right)} w_{\ell}^{2}=-\frac{1}{2} V i \frac{\partial \tilde{m}_{\ell}}{\partial(V i)} .
\end{gathered}
$$

After having made the variational principle (2) formally free from the constraint (1) by adding the left-hand side of Eq. (1) to $\Lambda_{\ell}$ by means of the Lagrange multiplier $\varphi_{\ell}$, we arrive at

$$
\delta \int_{t_{0}}^{t_{1}} d t \int_{x_{0}}^{x_{1}} \hat{\Lambda} / d x=0
$$

where

$$
\hat{\Lambda}_{\ell}=\Lambda_{\ell}+\varphi_{\ell}\left[\frac{\partial L}{\partial t}+\frac{\partial}{\partial x}(L v \ell)\right] .
$$

The Euler-Lagrange equations may be derived more easily from the equivalent Lagrangian density $\Lambda^{*}$ obtained from $\hat{\Lambda}_{\ell}$ by means of partial integration, viz.,

$$
\Lambda_{\ell}^{*}=\Lambda_{\ell}-L\left(\frac{\partial}{\partial t}+v_{\ell} \frac{\partial}{\partial x}\right) \varphi_{\ell} .
$$

Performing the variation of the internal variables $L$ and $w /$ in Eq. (24) yields the following Euler-Lagrange equations:

$$
\begin{gathered}
\delta L: \quad\left(\frac{\partial}{\partial t}+v_{\ell} \frac{\partial}{\partial x}\right) \varphi_{\ell}+\hat{\mu}_{\ell}=0, \\
\delta w_{\ell}: \quad m_{\ell} w_{\ell}=L \frac{\partial \varphi_{\ell}}{\partial x} .
\end{gathered}
$$

The generalized line-length potential $\hat{\mu}_{/}$appearing in Eq. (27) is, in view of Eqs. (8), (15), (16), (19), (21), and (22), determined by 


$$
\begin{aligned}
\hat{\mu}_{\ell}= & \mu \_-\frac{\partial g_{\ell}}{\partial x}=\rho \kappa \beta_{v}-\frac{1}{4} \kappa^{2} \frac{1}{V i} \frac{\partial \tilde{m}_{\ell}}{\partial(V i)}+\rho_{s} \frac{\kappa^{2}}{L^{3}} \gamma_{\ell}\left(\frac{\partial L}{\partial x}\right)^{2} \\
& -\frac{\kappa^{2}}{L^{2}} \frac{\partial}{\partial x}\left(\rho_{s} \gamma_{\ell} \frac{\partial L}{\partial x}\right)
\end{aligned}
$$

It will be clear from Eqs. (13) and (15) that

$$
P_{\ell}=m_{\ell} w_{\ell}
$$

represents the impulse, or pseudomomentum, density of the vortex tangle. When the quantity $B$ is introduced by means of

$$
B=\frac{P_{\ell}}{L},
$$

we have according to Eqs. (28) and (31)

$$
B=\frac{\partial \varphi_{\ell}}{\partial x} .
$$

Note that $B$ represents the local impulse of the vortex tangle per unit vortex length. Differentiating the Euler-Lagrange equation (27) partially with respect to the spatial coordinate $x$ yields

$$
\frac{\partial B}{\partial t}+\frac{\partial}{\partial x}\left(v_{\ell} B+\hat{\mu}_{\ell}\right)=0
$$

We shall refer to Eq. (33) as the dynamic equation of a vortex tangle. The nondissipative dynamics of a tangle of quantized vortices is apparently governed by Eqs. (1) and (33).

It should be kept in mind that the effective mass density $m_{\ell}$ that enters Eq. (33) according to Eqs. (30) and (31) and which, in view of Eqs. (20) and (23), is given by

$$
m_{\ell}=\tilde{m}_{\ell}-\frac{1}{2} V i \frac{\partial \tilde{m}_{\ell}}{\partial(V i)},
$$

involves an as yet unspecified function of the Vinen number $V i$. The further specification of this function will follow from the analysis of the energy of the vortex tangle in the next section.

\section{ENERGY AND IMPULSE OF VORTEX TANGLE}

It is known from the two-fluid hydrodynamics of He II that, when the flow velocities $v$ and $v_{n}$ are small compared to the propagation velocities of first and second sound, the external parameters $\rho$ and $S$ may be considered, in a first order of approximation, as constant [incompressibility approximation; see Landau and Lifshitz (Ref. 12)]. When, in addition, in one-dimensional flow the boundary conditions are time independent, the conservation equations for mass and entropy imply that also $v$ and $v_{n}$ take values that are independent of position and time.

When the conditions just mentioned are fulfilled, the Lagrangian density $\Lambda^{*}$ is invariant with respect to translations in space and time. The application of Noether's invariance theorem then yields equations that express the conservation of energy and impulse. They are given, respectively, by [see, e.g., Logan (Ref. 13)]

$$
\begin{gathered}
\frac{\partial H_{\ell}}{\partial t}+\frac{\partial Q_{\ell}}{\partial x}=0, \\
\frac{\partial P_{\ell}}{\partial t}+\frac{\partial \Pi_{\ell}}{\partial x}=0,
\end{gathered}
$$

where the energy density $H_{\ell}$ and the energy flux $Q_{\ell}$ are expressed by

$$
H_{\ell}=\sum_{\sigma=1}^{3} \frac{\partial \Lambda_{\ell}^{*}}{\partial\left(\partial \psi_{\sigma} / \partial t\right)} \frac{\partial \psi_{\sigma}}{\partial t}-\Lambda_{\ell}^{*}
$$

and

$$
Q_{\ell}=\sum_{\sigma=1}^{3} \frac{\partial \Lambda_{\ell}^{*}}{\partial\left(\partial \psi_{\sigma} / \partial x\right)} \frac{\partial \psi_{\sigma}}{\partial t},
$$

while the impulse density $P_{\ell}$ and the impulse flux $\Pi_{\ell}$ are determined by

$$
P_{\ell}=-\sum_{\sigma=1}^{3} \frac{\partial \Lambda_{\ell}^{*}}{\partial\left(\partial \psi_{\sigma} / \partial t\right)} \frac{\partial \psi_{\sigma}}{\partial x}
$$

and

$$
\Pi_{\ell}=-\sum_{\sigma=1}^{3} \frac{\partial \Lambda_{\ell}^{*}}{\partial\left(\partial \psi_{\sigma} / \partial x\right)} \frac{\partial \psi_{\sigma}}{\partial x}+\Lambda_{\ell}^{*} .
$$

The field variables $\psi_{\sigma}(\sigma=1,2,3)$ represent the internal variables $L$ and $w_{\ell}$ of the vortex tangle and the Lagrange multiplier $\varphi_{\ell}$. By using Eqs. (13), (15), and (26)-(28) we obtain from Eqs. (37)-(40)

$$
\begin{gathered}
H_{\ell}=-L \frac{\partial \varphi_{\ell}}{\partial t}+L\left(\frac{\partial}{\partial t}+v_{\ell} \frac{\partial}{\partial x}\right) \varphi_{\ell}-\Lambda_{\ell} \\
=v_{\ell} m_{\ell}+U_{\ell}, \\
Q_{\ell}=-L v_{\ell} \frac{\partial \varphi_{\ell}}{\partial t}-g_{\ell} \frac{\partial L}{\partial t} \\
=L v_{\ell}\left(v_{\ell} \frac{\partial \varphi_{\ell}}{\partial x}+\hat{\mu}_{\ell}\right)-g_{\ell} \frac{\partial L}{\partial t} \\
=v_{\ell}^{2} m_{\ell} w_{\ell}+v_{\ell} L \hat{\mu}_{\ell}-g_{\ell} \frac{\partial L}{\partial t}, \\
P_{\ell}=L \frac{\partial \varphi_{\ell}}{\partial x}=m_{\ell} w_{\ell},
\end{gathered}
$$

and

$$
\begin{aligned}
\Pi_{\ell} & =L v_{\ell} \frac{\partial \varphi_{\ell}}{\partial x}+g_{\ell} \frac{\partial L}{\partial x}+\Lambda_{\ell}^{*} \\
& =L v_{\ell} \frac{\partial \varphi_{\ell}}{\partial x}-L\left(\frac{\partial}{\partial t}+v_{\ell} \frac{\partial}{\partial x}\right) \varphi_{\ell}+\Lambda_{\ell}+g_{\ell} \frac{\partial L}{\partial x} \\
& =v_{\ell} m_{\ell} w_{\ell}+L \hat{\mu}_{\ell}-U_{\ell}+g_{\ell} \frac{\partial L}{\partial x} .
\end{aligned}
$$


Expression (43) for the impulse density of a vortex tangle is already familiar from the variational analysis in the preceding section.

When a Galilean transformation is performed according to

$$
\begin{gathered}
H_{\ell}=v P_{\ell}+H_{\ell}^{\prime}, \\
Q_{\ell}=v \Pi_{\ell}+v H_{\ell}^{\prime}+Q_{\ell}^{\prime}, \\
P_{\ell}=P_{\ell}^{\prime}, \\
\Pi_{\ell}=v P_{\ell}^{\prime}+\Pi_{\ell}^{\prime},
\end{gathered}
$$

the conservation equations (35) and (36) take the form

$$
\begin{gathered}
\frac{d H_{\ell}^{\prime}}{d t}+\frac{\partial Q_{\ell}^{\prime}}{\partial x}=0, \\
\frac{d P_{\ell}^{\prime}}{d t}+\frac{\partial \Pi_{\ell}^{\prime}}{\partial x}=0,
\end{gathered}
$$

where $d / d t=\partial / \partial t+v \partial / \partial x$. Note that in view of Eqs. (41) (48)

$$
\begin{gathered}
H_{\ell}^{\prime}=m_{\ell} w_{\ell}^{2}+U_{\ell}, \\
Q_{\ell}^{\prime}=w_{\ell}\left(m_{\ell} w_{\ell}^{2}+L_{\mu_{\ell}}\right)-g_{\ell} \frac{d L}{d t}, \\
P_{\ell}^{\prime}=m_{\ell} w_{\ell}, \\
\Pi_{\ell}^{\prime}=m_{\ell} w_{\ell}^{2}+L_{\ell}-U_{\ell}+g_{\ell} \frac{\partial L}{\partial x} .
\end{gathered}
$$

It may be recognized that, unless $m_{\ell}(V i)$ represents a linear function, $H_{\ell}^{\prime}$ is a Legendre transform of $U_{\ell}$. In fact,

$$
H_{\ell}^{\prime}=w_{\ell} P_{\ell}+U_{\ell},
$$

while

$$
d U_{\ell}=\hat{\mu}_{\ell} d L-P \ell d w_{\ell}+\frac{\partial}{\partial x}\left(g_{\ell} d L\right)
$$

and, accordingly,

$$
d H_{\ell}^{\prime}=\hat{\mu}_{\ell} d L+w \ell d P_{\ell}+\frac{\partial}{\partial x}\left(g_{\ell} d L\right) .
$$

Equations (55) - (57), however, also apply in the singular case where $m_{\ell}$ is a linear function of $V i$ [cf. Eq. (73)]. Let us introduce another Legendre transform, viz., the superfluid turbulent pressure $p_{\ell}$ defined by

$$
p_{\ell}=L \hat{\mu}_{\ell}-U_{\ell} .
$$

It follows from Eqs. (31), (56), and (58) that

$$
\begin{aligned}
d p_{\ell} & =L d \hat{\mu}_{\ell}+P_{\ell} d w_{\ell}-\frac{\partial}{\partial x}\left(g_{\ell} d L\right) \\
& =L\left(d \hat{\mu}_{\ell}+B d w_{\ell}\right)-\frac{\partial}{\partial x}\left(g_{\ell} d L\right) .
\end{aligned}
$$

The impulse flux (54) may, in virtue of Eq. (58), be expressed by

$$
\Pi_{\ell}^{\prime}=m_{\ell} w_{\ell}^{2}+p_{\ell}+g_{\ell} \frac{\partial L}{\partial x} .
$$

It is easily verified by taking Eqs. (13), (26), (27), and (58) into account that, in accordance with the Clebsch-Bateman principle,

$$
p_{\ell}=\Lambda_{\ell}^{*} .
$$

The equations for the conservation of energy and impulse may be derived directly from the kinematic equation (1) and the dynamic equation (33) governing the evolution of a vortex tangle. To that end these last equations are brought in the form

$$
\begin{gathered}
\frac{d L}{d t}+\frac{\partial}{\partial x}(L w \ell)=0, \\
\frac{d B}{d t}+\frac{\partial}{\partial x}\left(w \ell B+\hat{\mu}_{\ell}\right)=0 .
\end{gathered}
$$

Since, according to Eqs. (31), (47), and (57)

$$
d H_{\ell}^{\prime}=\left(\hat{\mu}_{\ell}+w_{\ell} B\right) d L+L w \varnothing d B+\frac{\partial}{\partial x}\left(g_{\ell} d L\right)
$$

and

$$
d P^{\prime}=B d L+L d B,
$$

we have in virtue of Eqs. (52), (54), (59), and (60)

$$
\begin{aligned}
\frac{d H_{\ell}^{\prime}}{d t} & =\left(\hat{\mu}_{\ell}+w_{\ell} B\right) \frac{d L}{d t}+L w \ell \frac{d B}{d t}+\frac{\partial}{\partial x}\left(g_{\ell} \frac{d L}{d t}\right) \\
& =-\frac{\partial}{\partial x}\left[L w_{\ell}\left(w_{\ell} B+\hat{\mu}_{\ell}\right)-g_{\ell} \frac{d L}{d t}\right] \\
& =-\frac{\partial}{\partial x} Q_{\ell}^{\prime}
\end{aligned}
$$

and

$$
\begin{aligned}
\frac{d P_{\ell}^{\prime}}{d t} & =B \frac{d L}{d t}+L \frac{d B}{d t} \\
& =-\frac{\partial}{\partial x}(L w / B)-L \frac{\partial \hat{\mu}_{\ell}}{\partial x}-L B \frac{\partial w_{\ell}}{\partial x} \\
& =-\frac{\partial}{\partial x}\left(L w / B+p_{\ell}+g \ell \frac{\partial L}{\partial x}\right) \\
& =-\frac{\partial}{\partial x} \Pi_{\ell}^{\prime} .
\end{aligned}
$$

Equations (66) and (67) express, respectively, the conservation of energy and impulse in conformity with Eqs. (49) and (50).

We remark that in view of Eqs. (14) and (51) 


$$
H_{\ell}^{\prime}=m_{\ell} w_{\ell}^{2}+U_{\ell}=m_{\ell} w_{\ell}^{2}-\frac{1}{2} \tilde{m}_{\ell} w_{\ell}^{2}+\widetilde{U}_{\ell} .
$$

This observation proves to be crucial for the further development of our hydrodynamic theory of superfluid turbulence. In fact, the total energy of a vortex tangle is contained in the "microscopic" kinetic energy associated with the circulating motion of the superfluid around the core of the quantized vortices. It is accordingly required that

$$
H_{\ell}^{\prime}=\widetilde{U}_{\ell}
$$

so that due to Eq. (68)

$$
m_{\ell}=\frac{1}{2} \tilde{m}_{\ell} .
$$

By substituting Eq. (34) in (70) the following differential equation is obtained for the unknown function $\tilde{m}_{\ell}$ of the Vinen number $V i$ :

$$
V i \frac{\partial \tilde{m}_{\ell}}{\partial(V i)}-\tilde{m}_{\ell}=0 .
$$

The general solution of Eq. (71) reads

$$
\tilde{m}_{\ell}=2 \rho_{s} \beta V i,
$$

where $\beta$ denotes a dimensionless integration constant involving, in general, the external parameters $\rho, S$, and $w_{n}$. In practice it will, principally, be a function of the absolute temperature, e.g., through $\rho_{s} / \rho$. See in that connection Sec. $\mathrm{V}$, in particular, expression (119). It follows from Eqs. (11), (70), and (72) that

$$
m_{\ell}=\rho_{s} \beta \kappa L^{1 / 2} /\left|w_{\ell}\right| .
$$

This expression for $m_{\ell}$ implies that

$$
P_{\ell}=\rho \kappa \hat{\beta} L^{1 / 2} \operatorname{sgn} w_{\ell},
$$

where

$$
\hat{\beta}=\frac{\rho_{s}}{\rho} \beta .
$$

The modified coefficient $\hat{\beta}$ is, like $\beta$, a function of the absolute temperature. The property that expression (74) for the impulse density does not involve the absolute value of the relative drift velocity, seems to be characteristic for a vortex tangle. It is obviously related to the anisotropy of the effective-mass tensor in the three-dimensional case (see Ref. 2).

The Lagrangian density $\Lambda_{\ell}$ defined by Eq. (5) may, in virtue of Eqs. (7), (8), (70), and (73), be expressed in the form

$$
\begin{aligned}
\Lambda_{\ell}= & \rho_{s} \kappa \beta L^{1 / 2}\left|w_{\ell}\right|-\rho_{s} \frac{\kappa^{2}}{4 \pi} L \ln \left(\frac{c}{a_{0} L^{1 / 2}}\right) \\
& -\frac{1}{2} \rho_{s} \frac{\kappa^{2}}{L^{2}} \gamma_{\ell}\left(\frac{\partial L}{\partial x}\right)^{2} .
\end{aligned}
$$

The first term on the right-hand side of Eq. (76) represents a velocity-dependent potential density [see Goldstein (Ref. 14) where a velocity-dependent potential is called Schering's potential function; see also the discussion in Ref. 2]. Let us introduce the modified coefficient $\hat{\gamma}_{\ell}$ associated with dispersive effects according to

$$
\hat{\gamma}_{\ell}=\frac{\rho_{s}}{\rho} \gamma_{\ell} .
$$

By using Eqs. (17), (18), (75), and (77) expression (76) for $\Lambda$ may be brought in the form

$$
\Lambda_{\ell}=\rho \kappa \hat{\beta} L^{1 / 2}\left|w_{\ell}\right|-\rho \kappa \beta_{v} L-\rho_{s} \frac{\kappa^{2}}{8 \pi} L-\frac{1}{2} \rho \frac{\kappa^{2}}{L^{2}} \hat{\gamma} \ell\left(\frac{\partial L}{\partial x}\right)^{2} .
$$

In view of Eqs. (11), (29), (72), (74), (75), and (77) the generalized line-length potential $\hat{\mu}_{/}$may be expressed by

$$
\begin{aligned}
\hat{\mu}_{\ell}= & \rho \kappa \beta_{v}-\frac{1}{2} \rho \kappa \hat{\beta} L^{-1 / 2} \mid w /+\rho \frac{\kappa^{2}}{L^{3}} \hat{\gamma}_{\ell}\left(\frac{\partial L}{\partial x}\right)^{2} \\
& -\frac{\kappa^{2}}{L^{2}} \frac{\partial}{\partial x}\left(\rho \hat{\gamma}_{\ell} \frac{\partial L}{\partial x}\right) \\
= & \rho \kappa \beta_{v}-w / \frac{\partial P \ell}{\partial L}+\rho \frac{\kappa^{2}}{L^{3}} \hat{\gamma} \ell\left(\frac{\partial L}{\partial x}\right)^{2}-\frac{\kappa^{2}}{L^{2}} \frac{\partial}{\partial x}\left(\rho \hat{\gamma}_{\ell} \frac{\partial L}{\partial x}\right) .
\end{aligned}
$$

It follows from Eqs. (78) and (79) that

$$
\begin{aligned}
p_{\ell}= & L \hat{\mu}_{\ell}+\Lambda_{\ell}=\frac{1}{2} \rho \kappa L^{1 / 2}\left(\hat{\beta}\left|w_{\ell}\right|-\frac{\kappa}{4 \pi} \frac{\rho_{s}}{\rho} L^{1 / 2}\right) \\
& +\frac{1}{2} \rho \frac{\kappa^{2}}{L^{2}} \hat{\gamma}_{\ell}\left(\frac{\partial L}{\partial x}\right)^{2}-\frac{\kappa^{2}}{L} \frac{\partial}{\partial x}\left(\rho \hat{\gamma}_{\ell} \frac{\partial L}{\partial x}\right) .
\end{aligned}
$$

By using Eqs. (14), (58), and (70), expression (52) for the energy flux $Q_{\ell}^{\prime}$ may take the following equivalent forms:

$$
\begin{aligned}
Q_{\ell}^{\prime} & =w_{\ell}\left(m_{\ell} w_{\ell}^{2}+L \hat{\mu}_{\ell}\right)-g_{\ell} \frac{d L}{d t} \\
& =w_{\ell}\left(m_{\ell} w_{\ell}^{2}+U_{\ell}+p_{\ell}\right)-g_{\ell} \frac{d L}{d t} \\
& =w_{\ell}\left(\widetilde{U}_{\ell}+p_{\ell}\right)-g_{\ell} \frac{d L}{d t},
\end{aligned}
$$

where, in view of Eqs. (7), (8), (17), (18), and (77)

$$
\widetilde{U}_{\ell}=\rho \kappa \beta_{v} L+\rho_{s} \frac{\kappa^{2}}{8 \pi} L+\frac{1}{2} \rho \frac{\kappa^{2}}{L^{2}} \hat{\gamma} \ell\left(\frac{\partial L}{\partial x}\right)^{2} .
$$

Note that $\widetilde{U}_{\ell}=H_{\ell}^{\prime}$ according to Eq. (69). The quantity $U_{\ell}+p_{\ell}$ appearing in Eq. (81) may be considered as the extended enthalpy density of the vortex tangle.

At this point of the analysis it seems appropriate for a better understanding of our approach of superfluid turbulence to recapitulate what has been achieved and to indicate what still has to be done, in the sequel of the paper, to complete the theory.

All equations derived so far apply to a nondissipative vortex tangle. Such a vortex tangle, however, may be realized only at absolute zero, where the dynamics of the tangle is not affected by the presence of elementary excitations. The sta- 
tus of the nondissipative vortex-tangle equations, like Eq. (62) expressing the conservation of line length of the quantized vortices and Eq. (50) for the conservation of vortextangle impulse, is comparable to that of the Euler equations in classical hydrodynamics which express the conservation of mass, momentum, and entropy in an ideal fluid without viscosity. In fact, the vortex-tangle equations just mentioned were derived from a variational principle of Hamilton's type, while it is known that the Euler equations may be obtained by a similar procedure.

In classical fluid dynamics the Euler equations are extended to the dissipative Navier-Stokes equations in order to complete the theory by taking into account real phenomena like the viscous properties of the fluid. It is known that this extension can be performed systematically by applying methods of the thermodynamics of irreversible processes. In a similar way we will introduce in the next section dissipative terms in the nondissipative equations for a vortex tangle. These terms will prove to be essential for arriving at a system of evolution equations that is physically realistic. It will be shown in Sec. $\mathrm{V}$ that the well understood homogeneous case $^{6,7}$ is completely covered by these extended equations.

\section{DISSIPATIVE EFFECTS}

The system of nondissipative evolution equations for a tangle of quantized vortices comprising the kinematic equation (62) and the dynamic equation (63) is clearly equivalent to the system composed of the kinematic equation (62) and the impulse equation (50). This last system, however, is slightly more convenient as a starting point for the introduction of additional dissipative terms. After having been supplied with these terms it assumes the form

$$
\begin{gathered}
\frac{d L}{d t}+\frac{\partial}{\partial x}\left(L w_{\ell}\right)=r_{\ell}, \\
\frac{d P_{\ell}}{d t}+\frac{\partial}{\partial x}\left(w_{\ell} P_{\ell}+p_{\ell}+g_{\ell} \frac{\partial L}{\partial x}\right)=F_{s \ell}+F_{n \ell}+F_{\ell},
\end{gathered}
$$

where $r_{\ell}$ represents the density of the net production rate of line length of quantized vortices in the tangle, while $F_{s}$ and $F_{n \ell}$ denote, respectively, the densities of the forces that the pure liquid and the elementary excitations exert on the quantized vortices. The quantity $F_{\ell}$ is introduced in the impulse equation as the density of a force resulting from internal friction in the tangle. Exterior forces, like the ones that are associated with the pinning of quantized vortices at the wall of a capillary, are not considered. Note that a possible diffusive contribution $q_{\ell}$ to the line-length flux has not been taken into account in Eq. (83). In fact, the physical background of such a term is not completely understood. In addition, its appearance in Eq. (83) unnecessarily complicates the subsequent development of our simplified hydrodynamic theory of superfluid turbulence, in particular with a view to the special cases of homogeneous flow and internal equilibrium to be treated in the following sections. The diffusive flux $q_{\ell}$, however, does appear in the analysis of Ref. 2 (note in that connection Ref. 15). It plays, in addition, an essential part in the observations on superfluid turbulence fronts by van Beelen et al. ${ }^{16}$
It is easily recognized that Eqs. (83) and (84) imply the following dissipative form of the dynamic equation of a vortex tangle:

$\frac{d B}{d t}+\frac{\partial}{\partial x}\left(w_{\ell} B+\hat{\mu}_{\ell}\right)=\frac{1}{L}\left(F_{s \ell}+F_{n \ell}\right)-\frac{r_{\ell}}{L} B+\frac{1}{L} F_{\ell}$.

The three-dimensional analysis in Ref. 2 shows that the conservation of total momentum comprising the momentum of the pure liquid and the impulses of the elementary excitations and the vortex tangle, requires that

$$
F_{\ell}=-\frac{\partial \Pi_{\ell}^{*}}{\partial x}
$$

Note that $F_{s \ell}$ and $F_{n \ell}$ represent mutual-friction forces exerted, respectively, by the pure liquid and the elementary excitations on the vortex tangle. These forces are, accordingly, accompanied by reaction forces acting in the opposite direction on the environment of the vortex tangle (see Ref. 2).

In the expression for the density $R_{\ell}$ of the entropy production rate associated with the vortex tangle two combinations of bilinear terms do appear, viz. (cf. the general analysis in Ref. 2)

$$
-F_{s \ell}\left(v_{\ell}-v\right)-F_{n \ell}\left(v_{\ell}-v_{n}\right)
$$

and

$$
-\hat{\mu}_{\ell} r_{\ell}-\Pi_{\ell}^{*} \frac{\partial w_{\ell}}{\partial x}
$$

The thermodynamics of irreversible processes then teaches us that (see, e.g., de Groot and Mazur ${ }^{17}$ )

$$
\begin{aligned}
& \left(\begin{array}{c}
-F_{s \ell} \\
-F_{n \ell}
\end{array}\right)=\left(C_{i j}\right)\left(\begin{array}{c}
v_{\ell}-v \\
v_{\ell}-v_{n}
\end{array}\right), \\
& \left(\begin{array}{c}
-\hat{\mu}_{\ell} \\
-\Pi_{\ell}^{*}
\end{array}\right)=\left(D_{i j}\right)\left(\begin{array}{c}
r_{\ell} \\
\partial w_{\ell} / \partial x
\end{array}\right) .
\end{aligned}
$$

The matrix coefficients $C_{i j}$ and $D_{i j}(i, j=1,2)$ have to satisfy the Onsager reciprocity relations

$$
C_{12}=C_{21}, \quad D_{12}=D_{21} .
$$

The coefficients may be brought in the form

$$
\begin{gathered}
C_{i j}=\rho_{s} \kappa L \gamma_{i j} \quad(i, j=1,2), \\
D_{11}=\rho_{s} \kappa L^{-2} \delta_{11}, \\
D_{12}=\rho_{s} \kappa L^{-1} \delta_{12}, \\
D_{22}=\rho_{s} \kappa \delta_{22},
\end{gathered}
$$

where $\gamma_{i j}$ and $\delta_{i j}(i, j=1,2)$ are dimensionless quantities involving only the external parameters $\rho, S$, and possibly also $w_{n}$. It seems natural to assume that they depend, effectively, on the absolute temperature $T$, e.g., through $\rho_{s} / \rho$. That assumption is supported by expressions (119) - (123). We shall follow the notation in Ref. 1 by using $\gamma$ instead of $\delta_{11}$, i.e., 


$$
\gamma=\delta_{11}
$$

In the sequel frequent use will be made of the modified coefficients $\hat{\gamma}, \hat{\gamma}_{i j}$, and $\hat{\delta}_{i j}(i, j=1,2)$ determined by

$$
\hat{\gamma}=\frac{\rho_{s}}{\rho} \gamma, \quad \hat{\gamma}_{i j}=\frac{\rho_{s}}{\rho} \gamma_{i j}, \quad \hat{\delta}_{i j}=\frac{\rho_{s}}{\rho} \delta_{i j} \quad(i, j=1,2) .
$$

In addition, the quantity $\hat{q}$ will be employed defined by

$$
\hat{q}=\frac{\hat{\gamma}_{12}+\hat{\gamma}_{22}}{\hat{\gamma}_{11}+2 \hat{\gamma}_{12}+\hat{\gamma}_{22}} \text {. }
$$

The mutual-friction force density $F_{s /}+F_{n \ell}$ appearing in Eqs. (84) and (85) may then, in view of Eq. (89), be written as

$$
F_{s \ell}+F_{n \ell}=\rho \kappa L\left(\hat{\gamma}_{11}+2 \hat{\gamma}_{12}+\hat{\gamma}_{22}\right)\left(\hat{q} w_{n}-w_{\ell}\right) .
$$

The requirement that the entropy production be non-negative implies that

$$
\begin{gathered}
\hat{\gamma}_{11} \geqslant 0, \quad \hat{\gamma}_{22} \geqslant 0, \quad \hat{\gamma}_{11} \hat{\gamma}_{22}-\hat{\gamma}_{12}^{2} \geqslant 0, \\
\hat{\gamma}=\hat{\delta}_{11} \geqslant 0, \quad \hat{\delta}_{22} \geqslant 0, \quad \hat{\delta}_{11} \hat{\delta}_{22}-\hat{\delta}_{12}^{2} \geqslant 0 .
\end{gathered}
$$

It follows from Eq. (97) that

$$
\begin{aligned}
& \hat{\gamma}_{11}+2 \hat{\gamma}_{12}+\hat{\gamma}_{22} \geqslant\left(\hat{\gamma}_{11}^{1 / 2}-\hat{\gamma}_{22}^{1 / 2}\right)^{2} \geqslant 0, \\
& \hat{\delta}_{11}+2 \hat{\delta}_{12}+\hat{\delta}_{22} \geqslant\left(\hat{\delta}_{11}^{1 / 2}-\hat{\delta}_{22}^{1 / 2}\right)^{2} \geqslant 0 .
\end{aligned}
$$

In view of Eqs. (57), (83), and (84) we have

$$
\begin{aligned}
\frac{d H_{\ell}^{\prime}}{d t}= & \hat{\mu}_{\ell} \frac{d L}{d t}+w \ell \frac{d P_{\ell}}{d t}+\frac{\partial}{\partial x}\left(g_{\ell} \frac{d L}{d t}\right) \\
= & -\frac{\partial}{\partial x}\left[w_{\ell}\left(L \hat{\mu}_{\ell}+P_{\ell} w_{\ell}\right)-g_{\ell} \frac{d L}{d t}+w_{\ell} \Pi_{\ell}^{*}\right] \\
& +\hat{\mu}_{\ell} r_{\ell}+\left(F_{s \ell}+F_{n \ell}\right) w_{\ell}+\Pi_{\ell}^{*} \frac{\partial w_{\ell}}{\partial x} .
\end{aligned}
$$

Accordingly, by taking account of Eq. (52),

$$
\frac{d H_{\ell}^{\prime}}{d t}+\frac{\partial}{\partial x}\left(Q_{\ell}^{\prime}+w \Pi_{\ell}^{*}\right)=F_{n}\left(v_{n}-v\right)-R_{\ell},
$$

where the density $R_{\ell}$ of the dissipation rate is given by [see Eqs. (87) and (88)]

$$
R_{\ell}=-\hat{\mu}_{\ell} r_{\ell}-\Pi_{\ell}^{*} \frac{\partial w_{\ell}}{\partial x}-F_{s \ell}(v \ell-v)-F_{n \ell}\left(v_{\ell}-v_{n}\right) .
$$

Equation (100) shows that, per unit volume, some part of the power $F_{n}\left(v_{n}-v\right)$ delivered by the mutual-force density $F_{n \ell}$ is being used for modifying the energy of the vortex tangle, while the rest of it is being dissipated. It should be noticed that, whereas, in a first order of approximation, the external parameters $\rho, S, v$, and $v_{n}$ and, therefore, also the absolute temperature $T$ take fixed values independent of position and time, the temperature gradient is, in a second order of approximation, determined by (see Ref. 1)

$$
S \frac{\partial T}{\partial x}=F_{\ell n}+F_{n}
$$

where $F_{\ell n}=-F_{n \ell}$, while $F_{n}$ represents the density of an exterior force acting on the elementary excitations, e.g., the one resulting from the no-slip of the elementary excitations at the wall of a capillary which leads, at laminar conditions, to Poiseuille's law.

\section{HOMOGENEOUS SUPERFLUID TURBULENCE AND VINEN'S EQUATION}

In the homogeneous case, when the spatial derivatives of the superfluid turbulent flow fields $L$ and $w_{\ell}$ vanish, the kinematic equation (83) reduces, in view of Eqs. (79), (90), and $(92)-(94)$, to the ordinary differential equation

$$
\frac{d L}{d t}=(1 / \hat{\gamma})\left[(\hat{\beta} / 2) L^{3 / 2} \mid w /-\beta_{v} L^{2}\right]
$$

The evolution equation (84) for the impulse density of the vortex tangle takes under homogeneous flow conditions, in virtue of Eqs. (74) and (96), the form

$$
\frac{d}{d t}\left(L^{1 / 2} \operatorname{sgn} w_{\ell}\right)=(1 / \hat{\beta}) L\left(\hat{\gamma}_{11}+2 \hat{\gamma}_{12}+\hat{\gamma}_{22}\right)\left(\hat{q} w_{n}-w_{\ell}\right) .
$$

It will be clear that $\hat{\beta}$ may be treated as a constant because the external parameters have, in a first order of approximation, values that are independent of position and time. Multiplying Eq. (104) by $2 L^{1 / 2} \operatorname{sgn} w /$ yields

$$
\frac{d L}{d t}=(2 / \hat{\beta})\left(\hat{\gamma}_{11}+2 \hat{\gamma}_{12}+\hat{\gamma}_{22}\right) L^{3 / 2}\left(\hat{q} w_{n} \operatorname{sgn} w_{\ell}-\left|w_{\ell}\right|\right) .
$$

Since the differential equations (103) and (105) are valid simultaneously, their right-hand sides should be equal. This implies that

$$
\begin{aligned}
& (2 / \hat{\beta})\left[\left(\hat{\beta}^{2} / 4 \hat{\gamma}\right)+\hat{\gamma}_{11}+2 \hat{\gamma}_{12}+\hat{\gamma}_{22}\right] L^{3 / 2}\left|w_{\ell}\right| \\
& \quad=(1 / \hat{\gamma}) \beta_{v} L^{2}+(2 / \hat{\beta})\left(\hat{\gamma}_{11}+2 \hat{\gamma}_{12}+\hat{\gamma}_{22}\right) L^{3 / 2} \hat{q} w_{n} \operatorname{sgn} w_{\ell} .
\end{aligned}
$$

Accordingly

$w_{\ell}=(1 / \hat{\Gamma})\left[\left(\hat{\gamma}_{12}+\hat{\gamma}_{22}\right) w_{n}+(\hat{\beta} / 2 \hat{\gamma}) \beta_{v} L^{1 / 2} \operatorname{sgn} w_{\ell}\right]$,

where

$$
\hat{\Gamma}=\left(\hat{\beta}^{2} / 4 \hat{\gamma}\right)+\hat{\gamma}_{11}+2 \hat{\gamma}_{12}+\hat{\gamma}_{22} \text {. }
$$

By taking Eqs. (95), (97), and (98) into account it follows from Eq. (107) that

$$
\epsilon\left(\hat{\gamma}_{12}+\hat{\gamma}_{22}\right)\left|w_{n}\right|+(\hat{\beta} / 2 \hat{\gamma}) \beta_{v} L^{1 / 2} \geqslant 0
$$

where

$$
\epsilon=\operatorname{sgn} w_{\ell} \operatorname{sgn} w_{n}= \pm 1
$$

Substitution of Eq. (107) in either Eq. (103) or Eq. (105) produces the differential equation 


$$
\begin{aligned}
\frac{d L}{d t}= & (\hat{\beta} / 2 \hat{\gamma})(1 / \hat{\Gamma})\left(\hat{\gamma}_{12}+\hat{\gamma}_{22}\right) \epsilon\left|w_{n}\right| L^{3 / 2} \\
& -(1 / \hat{\gamma})(1 / \hat{\Gamma})\left(\hat{\gamma}_{11}+2 \hat{\gamma}_{12}+\hat{\gamma}_{22}\right) \beta_{v} L^{2} .
\end{aligned}
$$

Let us introduce the quantities $\chi_{1}$ and $\chi_{2}$ according to

$$
\chi_{1} \alpha=(\hat{\beta} / 2 \hat{\gamma})(1 / \hat{\Gamma})\left(\hat{\gamma}_{12}+\hat{\gamma}_{22}\right)\left(\rho_{s} / \rho\right)
$$

and

$$
(\kappa / 2 \pi) \chi_{2}=(1 / \hat{\gamma})(1 / \hat{\Gamma})\left(\hat{\gamma}_{11}+2 \hat{\gamma}_{12}+\hat{\gamma}_{22}\right) \beta_{v} .
$$

The coefficient $\alpha$ appearing in Eq. (112) is used by Schwarz. ${ }^{7}$ It is related to the first Hall-Vinen coefficient $B$ by means of

$$
\alpha=\left(\rho_{n} / 2 \rho\right) B=\left(\rho_{s} / \rho\right) \alpha_{v} .
$$

The coefficient $\alpha_{v}$ has been introduced in Ref. 3. Corresponding relations with regard to the second Hall-Vinen coefficient read

$$
1-\alpha^{\prime}=1-\left(\rho_{n} / 2 \rho\right) B^{\prime}=\left(\rho_{s} / \rho\right)\left(1-\alpha_{v}^{\prime}\right) .
$$

By using Eqs. (112) and (113) and realizing that $v_{n}-v=\left(\rho_{s} / \rho\right)\left(v_{n}-v_{s}\right)$ the following slightly generalized form of Vinen's equation is obtained from Eq. (111):

$$
\frac{d L}{d t}=\chi_{1} \alpha \epsilon\left|v_{n}-v_{s}\right| L^{3 / 2}-(\kappa / 2 \pi) \chi_{2} L^{2} .
$$

When $\epsilon=1$, it reduces to the Vinen equation discussed by Schwarz. ${ }^{7}$ It should be noticed that, in view of Eq. (113), the quantity $\chi_{2}$ is, like $\beta_{v}$, logarithmically dependent on $L$ [see Eq. (17)]. This weak dependence on $L$ is usually disregarded in applications of Vinen's equation. It was also ignored by Vinen in his seminal papers. ${ }^{18}$ It should be emphasized that the Vinen equation (116) being valid for homogeneous flow conditions was derived by combining the two equations (103) and (104) governing the evolution of a homogeneous vortex tangle. By the same procedure the algebraic expression (107) was obtained for the relative drift velocity of the vortex tangle. The question has been raised how the single Vinen equation (116) might be generalized to inhomogeneous flow conditions. That problem, however, is in the light of our hydrodynamic theory of superfluid turbulence not properly posed. In fact, the Vinen equation (116) for the time evolution of $L$ and expression (107) for $w /$ should not be considered separately; they are intimately related within the context of the present theory. The generalization to the inhomogeneous case is, accordingly, obvious: the system of equations (83) and (84) generalizes the set of equations (107) and (116).

By substituting Eq. (107) in Eq. (89) and applying Eqs. (92)-(94) the following expressions for the mutual-friction forces may be derived:

$$
\begin{gathered}
F_{n \ell} / \rho \kappa L=(1 / \hat{\Gamma})\left\{\left[\left(\hat{\beta}^{2} / 4 \hat{\gamma}\right) \hat{\gamma}_{22}+\hat{\gamma}_{11} \hat{\gamma}_{22}-\hat{\gamma}_{12}^{2}\right] w_{n}\right. \\
\left.-(\hat{\beta} / 2 \hat{\gamma})\left(\hat{\gamma}_{12}+\hat{\gamma}_{22}\right) \beta_{v} L^{1 / 2} \operatorname{sgn} w_{\ell}\right\}, \\
F_{s \ell} / \rho \kappa L=\left(1 / \hat{\Gamma}\left\{\left[\left(\hat{\beta}^{2} / 4 \hat{\gamma}\right) \hat{\gamma}_{12}-\left(\hat{\gamma}_{11} \hat{\gamma}_{22}-\hat{\gamma}_{12}^{2}\right)\right] w_{n}\right.\right. \\
\left.-(\hat{\beta} / 2 \hat{\gamma})\left(\hat{\gamma}_{11}+\hat{\gamma}_{12}\right) \beta_{v} L^{1 / 2} \operatorname{sgn} w \ell\right\} .
\end{gathered}
$$

Schwarz ${ }^{7}$ has shown that an averaging procedure with respect to the quantized vortices in a homogeneous vortex tangle yields definite expressions for $w_{\ell}, d L / d t$, and $F_{n \ell}$. The averaging may be based (see Ref. 3) on a generalized "microscopic" equation of motion for a line element of a quantized vortex in which, in addition to the modified HallVinen coefficients $\alpha_{v}$ and $\alpha_{v}^{\prime}$ the coefficient $\alpha_{v}{ }^{\prime \prime}$ enters. This coefficient determines the tangential component of the local velocity of the line vortex. It should be realized that the coefficient $\alpha_{v}$ " is not related to the third Hall-Vinen coefficient $B^{\prime \prime}$ considered, e.g., in Donnelly. ${ }^{19}$ This last coefficient which determines the tangential component of the local force acting on the line vortex is usually taken equal to zero (see the argument in Swanson and Donnelly $\left.{ }^{20}\right)$.

By equating expressions (107), (116), and (117) for, respectively, $w_{\ell}, d L / d t$, and $F_{n \ell}$ to the corresponding expressions that have been obtained by averaging over the quantized vortices in a tangle we arrive at the identities (see Ref. 3)

$$
\begin{gathered}
\hat{\beta} / 2 c_{L}=\left[I_{\|}+\alpha_{v}^{\prime \prime}\left(1-I_{\|}\right)-\left(1-\alpha_{v}^{\prime}\right)\left(I_{\|}-I_{\ell} c_{L}\right)\right]^{-1}, \\
2 \hat{\gamma} / \hat{\beta}=\left(\alpha_{v} I_{\ell}\right)^{-1}\left[I_{\|}+\alpha_{v}^{\prime \prime}\left(1-I_{\|}\right)-\left(1-\alpha_{v}^{\prime}\right) I_{\|}\right],
\end{gathered}
$$

$$
\begin{gathered}
\hat{\gamma}_{11}=\alpha_{v}\left(1-\alpha_{v}^{\prime}\right)^{-1}\left[I_{\|}+\alpha_{v}^{\prime \prime}\left(1-I_{\|}\right)\right]^{-1}\left\{\left(1-\alpha_{v}^{\prime \prime}\right)^{2}\left(1-I_{\|}\right)^{2}\right. \\
\left.-1+\left(\hat{\beta} / 2 c_{L}\right)\left[I_{\|}+\alpha_{v}^{\prime \prime}\left(1-I_{\|}\right)\right]\right\} \\
\hat{\gamma}_{12}=\alpha_{v}\left(1-\alpha_{v}^{\prime}\right)^{-1}\left\{1-\left[I_{\|}+\alpha_{v}^{\prime \prime}\left(1-I_{\|}\right)\right]\right\} \\
\hat{\gamma}_{22}=\alpha_{v}\left(1-\alpha_{v}^{\prime}\right)^{-1}\left[I_{\|}+\alpha_{v}^{\prime \prime}\left(1-I_{\|}\right)\right] .
\end{gathered}
$$

The quantities $I_{\|}, I_{\ell}$, and $c_{L}$ appearing in Eqs. (119)-(123) represent coefficients that have been introduced by Schwarz. ${ }^{7}$ These coefficients are defined as definite averages over the quantized vortices in a tangle (see also Ref. 3). Equations (119) - (123), obviously, express the five macroscopic coefficients $\hat{\beta}, \hat{\gamma}, \hat{\gamma}_{i j}(i, j=1,2)$, note the reciprocity relation, in terms of the six quantities $\alpha_{v}, \alpha_{v}^{\prime}, \alpha_{v}^{\prime \prime}, I_{\|}$, $I_{\ell}$, and $c_{L}$. The macroscopic coefficients, however, have to fulfill the dissipative inequalities (97). In Appendix B of Ref. 8 it is demonstrated that in the case where $1-\alpha_{v}^{\prime} \geqslant 0$ or, equivalently, $1-\alpha^{\prime} \geqslant 0$, inequalities that are known to be satisfied within a relatively large range of absolute temperatures (see Ref. 9), the dissipative inequalities for $\hat{\gamma}$ and $\hat{\gamma}_{i j}$ $(i, j=1,2)$ are fulfilled if and only if

$$
\alpha_{v}^{\prime \prime} \geqslant-\alpha_{v}^{\prime} I_{\|}\left(1-I_{\|}\right)^{-1}
$$

or, equivalently,

$$
\alpha^{\prime \prime} \geqslant\left(\frac{\rho_{n}}{\rho}-\alpha^{\prime} I_{\|}\right)\left(1-I_{\|}\right)^{-1},
$$

where $1-\alpha^{\prime \prime}=\left(\rho_{s} / \rho\right)\left(1-\alpha_{v}^{\prime \prime}\right)$. In the case of permanent internal equilibrium, i.e., when $\hat{\gamma}=0$ (see the next section), the equality sign holds in Eqs. (124) and (125). That property is easily verified by means of Eq. (120). 


\section{INTERNAL EQUILIBRIUM AND BURGERS' EQUATION WITH NONLINEAR SOURCE TERM}

In this section it is assumed that the cross effects associated with the macroscopic coefficients $\hat{\delta}_{12}$ and $\hat{\delta}_{21}$ may be disregarded, i.e., we take

$$
\hat{\delta}_{12}=\hat{\delta}_{21}=0 .
$$

The following terminology will be used: (1) the vortex tangle is in internal equilibrium if and only if

$$
\hat{\mu}_{\ell}=0
$$

(ii) the vortex tangle is in external equilibrium with the pure liquid and the elementary excitations if and only if

$$
F_{s \ell}+F_{n \ell}=0 \text {. }
$$

It will be clear that in the homogeneous case external equilibrium entails internal equilibrium. In fact, in the case of external equilibrium the right-hand side of Eq. (105) vanishes. Accordingly $d L / d t=0$ so that, by virtue of the kinematic equation (103) and Eq. (79), the vortex tangle is in internal equilibrium. ${ }^{21}$ The converse statement is also true unless $\hat{\gamma}=0$. In fact, when $\hat{\gamma}$ vanishes the vortex tangle may be in internal equilibrium without satisfying condition (128) for external equilibrium. The case $\hat{\gamma}=0$, which may be of substantial importance in practice, will be investigated in this section in more detail. Note that the requirement $\hat{\gamma}=0$ entails, in view of the dissipative inequalities (97), condition (126).

When $\hat{\gamma}$ vanishes in the general inhomogeneous case, it follows immediately from Eqs. (90), (92) - (94), and (126) that condition (127) for internal equilibrium of the vortex tangle is fulfilled permanently. This implies, in view of Eq. (79), that

$$
\begin{aligned}
\left|w_{\ell}\right|= & \left(2 \beta_{v} / \hat{\beta}\right) L^{1 / 2}+(2 \kappa / \hat{\beta})\left[L^{-5 / 2} \hat{\gamma}_{\ell}\left(\frac{\partial L}{\partial x}\right)^{2}\right. \\
& \left.-L^{-3 / 2} \frac{\partial}{\partial x}\left(\hat{\gamma}_{\ell} \frac{\partial L}{\partial x}\right)\right] .
\end{aligned}
$$

Substituting Eq. (129) in Eq. (80) yields the following expression for the superfluid turbulent pressure:

$$
\begin{aligned}
p_{\ell}= & \rho \kappa \beta_{v} L-\rho_{s} \frac{\kappa^{2}}{8 \pi} L+\frac{3}{2} \rho \frac{\kappa^{2}}{L^{2}} \hat{\gamma}\left(\frac{\partial L}{\partial x}\right)^{2} \\
& -2 \rho \frac{\kappa^{2}}{L} \frac{\partial}{\partial x}\left(\hat{\gamma}_{\ell} \frac{\partial L}{\partial x}\right) .
\end{aligned}
$$

In internal equilibrium relation (59) reduces to

$$
d p_{\ell}=P_{\ell} d w_{\ell}-\frac{\partial}{\partial x}\left(g_{\ell} d L\right)
$$

so that the impulse equation (84) is given by

$$
\frac{d P_{\ell}}{d t}+\frac{\partial}{\partial x}\left(w_{\ell} P_{\ell}\right)+P_{\ell} \frac{\partial w_{\ell}}{\partial x}=F_{s \ell}+F_{n \ell}+F_{\ell} .
$$

By taking Eqs. (74), (86), (90), (92), (94), (96), and (126) into account Eq. (132) may be brought in the form

$$
\begin{aligned}
& \frac{d}{d t}\left(\hat{\beta} L^{1 / 2} \operatorname{sgn} w_{\ell}\right)+w \ell \frac{\partial}{\partial x}\left(\hat{\beta} L^{1 / 2} \operatorname{sgn} w_{\ell}\right) \\
& +2 \hat{\beta} L^{1 / 2} \operatorname{sgn} w_{\ell} \frac{\partial w_{\ell}}{\partial x}=L\left(\hat{\gamma}_{11}+2 \hat{\gamma}_{12}+\hat{\gamma}_{22}\right)\left(\hat{q} w_{n}-w_{\ell}\right) \\
& \quad+\frac{\partial}{\partial x}\left(\hat{\delta}_{22} \frac{\partial w \ell}{\partial x}\right) .
\end{aligned}
$$

By substituting Eq. (129) in Eq. (133) and introducing the dependent variable $y$ according to

$$
y=L^{1 / 2} \operatorname{sgn} w /
$$

the following partial differential equation is obtained:

$$
\begin{aligned}
& \frac{d y}{d t}+\left[\left(2 \beta_{v} / \hat{\beta}\right) y+(8 \kappa / \hat{\beta}) \hat{\gamma}_{\ell} y^{-3}\left(\frac{\partial y}{\partial x}\right)^{2}-(4 \kappa / \hat{\beta}) \hat{\gamma}_{\ell} y^{-2} \frac{\partial^{2} y}{\partial x^{2}}\right] \frac{\partial y}{\partial x}+2 y \frac{\partial}{\partial x}\left[\left(2 \beta_{v} / \hat{\beta}\right) y+(8 \kappa / \hat{\beta}) \hat{\gamma}_{\ell} y^{-3}\left(\frac{\partial y}{\partial x}\right)^{2}\right. \\
&\left.-(4 \kappa / \hat{\beta}) \hat{\gamma}_{\ell} y^{-2} \frac{\partial^{2} y}{\partial x^{2}}\right]=(1 / \hat{\beta})\left(\hat{\gamma}_{11}+2 \hat{\gamma}_{12}+\hat{\gamma}_{22}\right) y^{2}\left[\hat{q} w_{n}-\left(2 \beta_{v} / \hat{\beta}\right) y-(8 \kappa / \hat{\beta}) \hat{\gamma}_{\ell} y^{-3}\left(\frac{\partial y}{\partial x}\right)^{2}+(4 \kappa / \hat{\beta}) \hat{\gamma}_{\ell y^{-2}} \frac{\partial^{2} y}{\partial x^{2}}\right] \\
&+\left(\hat{\delta}_{22} / \hat{\beta}\right) \frac{\partial^{2}}{\partial x^{2}}\left[\left(2 \beta_{v} / \hat{\beta}\right) y+(8 \kappa / \hat{\beta}) \hat{\gamma}_{\ell} y^{-3}\left(\frac{\partial y}{\partial x}\right)^{2}-(4 \kappa / \hat{\beta}) \hat{\gamma}_{\ell} y^{-2} \frac{\partial^{2} y}{\partial x^{2}}\right] .
\end{aligned}
$$

When dispersive effects are neglected, i.e., $\hat{\gamma}_{\ell}=0$ and, in addition, $\beta_{v}$ is treated as a constant, Eq. (135) reduces to

$$
\frac{d y}{d t}+\left(6 \beta_{v} / \hat{\beta}\right) y \frac{\partial y}{\partial x}=\hat{\delta}_{22}\left(2 \beta_{v} / \hat{\beta}^{2}\right) \frac{\partial^{2} y}{\partial x^{2}}+(1 / \hat{\beta})\left(\hat{\gamma}_{11}+2 \hat{\gamma}_{12}+\hat{\gamma}_{22}\right) y^{2}\left[\hat{q} w_{n}-\left(2 \beta_{v} / \hat{\beta}\right) y\right] .
$$


This equation represents Burgers' equation supplemented with a nonlinear source term. ${ }^{22}$ In the Appendix some exact solutions of Eq. (136) are listed. They may be considered as direct extensions, to the case where diffusive effects are taken into account, of the marginally stable superfluid turbulence fronts investigated in Ref. 8.

In permanent internal equilibrium $(\hat{\gamma}=0)$ the density $r_{\ell}$ of the net production rate of line length is, in view of Eqs. (83) and (129), determined by

$$
r_{\ell}=\frac{d L}{d t}+\frac{\partial}{\partial x}\left\{L\left[\left(2 \beta_{v} / \hat{\beta}\right) L^{1 / 2} \operatorname{sgn} w_{\ell}+(2 \kappa / \hat{\beta}) L^{-5 / 2} \hat{\gamma}_{\ell}\left(\frac{\partial L}{\partial x}\right)^{2} \operatorname{sgn} w_{\ell}-(2 \kappa / \hat{\beta}) L^{-3 / 2} \frac{\partial}{\partial x}\left(\hat{\gamma}_{\ell} \frac{\partial L}{\partial x}\right) \operatorname{sgn} w_{\ell}\right]\right\} .
$$

The superfluid turbulent pressure $p$ fulfills in internal equilibrium, according to Eqs. (58) and (127), the relation

$$
p_{\ell}=-U_{\ell} .
$$

Since, in view of Eq. (13), $-U_{\ell}=\Lambda_{\ell}$, this property is reminiscent of the Clebsch-Bateman principle (61). Its range of validity, however, is both wider, it applies to dissipative flow processes, and smaller, it is restricted to internal equilibrium.

We finally remark that internal equilibrium is associated with the dissipative limit $(\hat{\gamma} \rightarrow 0)$ of the balance equation of line length (83), while conservation of line length corresponds to the nondissipative limit $(\hat{\gamma} \rightarrow \infty)$ of this equation.

\section{REVIEW OF BASIC EQUATIONS}

Since many equations have passed in review in the preceding sections, it seems appropriate, for a right appreciation of our simplified hydrodynamic theory of superfluid turbulence, to list here the most relevant equations in their most accessible form for the particular but important case where dispersive effects are disregarded $\left(\hat{\gamma}_{\ell}=0\right)$. The cross effects that are associated with the coefficients $\hat{\delta}_{12}$ and $\hat{\delta}_{21}$ will also be neglected. In that connection it should be realized that, in general, cross effects are relatively small.

A simple analysis using Eqs. (79), (90), (92), and (93) shows that the kinematic equation (83) of a vortex tangle may be represented by

$$
\frac{d y}{d t}+w_{\ell} \frac{\partial y}{\partial x}+\frac{1}{2} y \frac{\partial w_{\ell}}{\partial x}=(\hat{\beta} / 4 \hat{\gamma}) y^{2}\left[w_{\ell}-\left(2 \beta_{v} / \hat{\beta}\right) y\right]
$$

where the dependent variable $y$ is related directly to the linelength density $L$ according to $y=L^{1 / 2} \operatorname{sgn} w_{/}$[ [see expression (134) of the preceding section]. In a similar way, by applying Eqs. (74), (80), (86), (90), (92), (94),(96), and (134) the impulse equation (84), or dynamic equation (85), takes the form

$$
\begin{aligned}
\frac{d y}{d t}+ & \frac{\partial}{\partial x}\left[\frac{3}{2} y w_{\ell}-(1 / \hat{\beta}) \frac{\kappa}{8 \pi} \frac{\rho_{s}}{\rho} y^{2}\right] \\
= & (1 / \hat{\beta})\left(\hat{\gamma}_{11}+2 \hat{\gamma}_{12}+\hat{\gamma}_{22}\right) y^{2}\left(\hat{q} w_{n}-w_{\ell}\right) \\
& +\left(\hat{\delta}_{22} / \hat{\beta}\right) \frac{\partial^{2} w_{\ell}}{\partial x^{2}} .
\end{aligned}
$$

The second term between square brackets on the left-hand side of this equation has to be suppressed when the quantity $\beta_{v}$, which depends, in view of Eq. (17), logarithmically on $|y|$, is being treated as a constant. In fact, in that case the dimensionless constants $c$ and $c^{\prime}$ introduced, respectively, by Eqs. (7) and (19) should not be distinguished, so that relation (18) has to be skipped. By eliminating the time derivative between Eqs. (139) and (140) a spatial differential equation is obtained that couples the ways in which $y$ and $w_{\ell}$ depend on position.

In the special cases that have been considered in Secs. V and VI the system of equations (139) and (140) takes a particularly simple form.

(i) Homogeneous case. It is easily verified (see the analysis in Sec. V) that, when homogeneous flow conditions prevail in a vortex tangle, the system of equations (139) and (140) is equivalent to an algebraic equation for $w_{/}$, viz.,

$$
w_{\ell}=(1 / \hat{\Gamma})\left(\hat{\gamma}_{12}+\hat{\gamma}_{22}\right) w_{n}+(1 / \hat{\Gamma})(\hat{\beta} / 2 \hat{\gamma}) \beta_{v} y
$$

and an ordinary differential equation (generalized Vinen equation) for $y$ given by

$$
\begin{aligned}
\frac{d y}{d t}= & (\hat{\beta} / 4 \hat{\gamma})(1 / \hat{\Gamma})\left(\hat{\gamma}_{12}+\hat{\gamma}_{22}\right) w_{n} y^{2} \\
& -(1 / 2 \hat{\gamma})(1 / \hat{\Gamma})\left(\hat{\gamma}_{11}+2 \hat{\gamma}_{12}+\hat{\gamma}_{22}\right) \beta_{v} y^{3} .
\end{aligned}
$$

Note that $\hat{\Gamma}$ is defined by Eq. (108). When written in terms of the Schwarz coefficients $I_{\|}, I_{\ell}$, and $c_{L}$, Eqs. (141) and (142) take, respectively, the form

$$
\begin{gathered}
w_{\ell}=\left[\alpha_{v}^{\prime} I_{\|}+\alpha_{v}^{\prime \prime}\left(1-I_{\|}\right)\right] w_{n}+\left(1-\alpha_{v}^{\prime}\right) I_{\ell} \beta_{v} y, \\
\frac{d y}{d t}=\frac{1}{2} \alpha_{v} I_{\ell} y^{2}\left[w_{n}-\left(\beta_{v} / c_{L}\right) y\right] .
\end{gathered}
$$

(ii) Internal equilibrium. When the vortex tangle is in permanent internal equilibrium $(\hat{\gamma}=0)$, the right-hand side of Eq. (139) vanishes so that

$$
w_{\ell}=\left(2 \beta_{v} / \hat{\beta}\right) y .
$$

Substitution of this expression for $w /$ in Eq. (140) yields Eq. (136) which constitutes a generalized form of Burgers' equation. Note that in the derivation of this equation the quantity $\beta_{v}$ has been treated as a constant. When Eqs. (145) and (136) are written in terms of the Schwarz coefficients, they take, respectively, the form

$$
w_{\ell}=\left(1-\alpha_{v}^{\prime}\right) I_{\ell} \beta_{v} y,
$$




$$
\begin{aligned}
\frac{d y}{d t}+3\left(1-\alpha_{v}^{\prime}\right) I \beta_{v} y \frac{\partial y}{\partial x}= & \hat{\delta}_{22} \frac{1}{2}\left[\left(1-\alpha_{v}^{\prime}\right) I_{\ell}\right]^{2} \beta_{v} \frac{\partial^{2} y}{\partial x^{2}} \\
& +\frac{1}{2} \alpha_{v} I_{\ell} y^{2}\left[w_{n}-\left(\beta_{v} / c_{L}\right) y\right]
\end{aligned}
$$

It will be clear that in the case of homogeneous flow, Eq. (147) passes into the generalized Vinen equation (144). The form of the Vinen equation is, accordingly, not affected explicitly by the condition of internal equilibrium (see also the discussion in Ref. 8). Note, however, that, in view of Eq. (120), the coefficient of $w_{n}$ in Eq. (143) vanishes when $\hat{\gamma}=0$, so that the expression for the relative drift velocity of a homogeneous vortex tangle reduces, in the case of permanent internal equilibrium, to the more simple form Eq. (146). It may be concluded that under some slightly restricting conditions, viz., when internal equilibrium prevails and dispersive effects are negligible, Eq. (147), or similarly Eq. (136), may be considered as an extension of Vinen's equation to inhomogeneous superfluid turbulence.

We finally list some conclusions.

(1) It has been proved possible to derive from a few basic principles a single partial differential equation for the linelength density $L(x, t)$ of inhomogeneous superfluid turbulence that generalizes Vinen's equation. In the case where Vinen's equation takes, according to Schwarz, ${ }^{7}$ the form (144), the partial differential equation is given by Eq. (147).
Note that the dependent variable $y$ is according to Eq. (134), irrespective of the sign of the relative drift velocity $w_{\ell}$ of the vortex tangle, equal to the square root of the line-length density.

(2) Equation (147) has been obtained from the impulse equation (84) for a vortex tangle by making a few simplifying assumptions, viz., absence of dispersive effects and realization of internal equilibrium of the vortex tangle. These assumptions are expected to be fulfilled in many cases of practical interest.

(3) An interesting new contribution to the internal dynamics of an inhomogeneous vortex tangle is supplied by the first term on the right-hand side of Eq. (147) which models the diffusion of vortex-tangle impulse.

(4) In developing the theory a quantity $p_{\ell}$ called superfluid turbulent pressure could be introduced. Its explicit expression as a function of $L$ and $w_{\ell}$ is given by Eq. (80). When the vortex tangle is in internal equilibrium, the superfluid turbulent pressure satisfies Eq. (138).

\section{APPENDIX: SOME EXACT SOLUTIONS OF EQ. (136) REPRESENTING SUPERFLUID TURBULENCE FRONTS}

Equation (136) which, as shown in Sec. VI, directly follows from the impulse equation (84) for a vortex tangle in the physically realistic case where dispersive effects may be neglected and the vortex tangle is in internal equilibrium, reads $\left(y=L^{1 / 2} \operatorname{sgn} w_{\ell}\right)$

$$
\frac{d y}{d t}+\left(6 \beta_{v} / \hat{\beta}\right) y \frac{\partial y}{\partial x}=\hat{\delta}_{22}\left(2 \beta_{v} / \hat{\beta}^{2}\right) \frac{\partial^{2} y}{\partial x^{2}}+(1 / \hat{\beta})\left(\hat{\gamma}_{11}+2 \hat{\gamma}_{12}+\hat{\gamma}_{22}\right) y^{2}\left[\hat{q} w_{n}-\left(2 \beta_{v} / \hat{\beta}\right) y\right]
$$

Note that the first term on the right-hand side of Eq. (136) models the diffusion of vortex-tangle impulse, while the second term accounts for the mutual friction like in Vinen's equation (144). We are looking for solutions of Eq. (136) that represent propagating waves of permanent form. We accordingly take

$$
y=y\left[x-x_{0}-v_{f}\left(t-t_{0}\right)\right] .
$$

In that case

$$
\frac{d y}{d t}=-w_{f} \frac{\partial y}{\partial x},
$$

where

$$
w_{f}=v_{f}-v .
$$

Let us introduce the following parameters (see Ref. 8):

$$
\begin{gathered}
b=\left(\hat{\beta} / 2 \beta_{v}\right) \hat{q} w_{n}, \\
A=\left(6 \beta_{v} / w_{n}\right)\left(\hat{\gamma}_{12}+\hat{\gamma}_{22}\right)^{-1}, \\
B=w_{f}\left(3 \hat{q} w_{n}\right)^{-1},
\end{gathered}
$$

$$
C=\hat{\delta}_{22}\left(2 \beta_{v} / \hat{\beta}\right)^{2}\left(\hat{\gamma}_{12}+\hat{\gamma}_{22}\right)^{-1}\left(\hat{q} w_{n}^{2}\right)^{-1} .
$$

These parameters may be expressed in terms of the Schwarz coefficients by using Eqs. (119) - (123). Since Eq. (136) is valid provided $\hat{\gamma}=0$ (permanent internal equilibrium), the expressions in terms of the Schwarz coefficients should also apply to that particular case. They are given by

$$
b=\left(c_{L} / \beta_{v}\right) w_{n},
$$

$$
\begin{aligned}
& A=\left(6 \beta_{v} / w_{n}\right)\left(1-\alpha_{v}^{\prime}\right) \alpha_{v}^{-1} \\
& B=w_{f}\left[3\left(1-\alpha_{v}^{\prime}\right) I / c_{L} w_{n}\right]^{-1} \\
& C / A^{2}=\hat{\delta}_{22}(1 / 36)\left(\alpha_{v} I / c_{L}\right)
\end{aligned}
$$

Whereas the parameter $B$ is clearly dimensionless, the quantities $b, A$, and $C$ have the dimension of, respectively, reciprocal length, length, and length squared. It seems therefore natural to introduce dimensionless variables $\eta$ and $\xi$ according to

$$
\eta=y / b, \quad \xi=x / A .
$$


By applying the dimensional scaling (A12), Eq. (136) passes, in view of Eq. (A2), into

$$
(\eta-B) \frac{\partial \eta}{\partial \xi}=\left(C / A^{2}\right) \frac{\partial^{2} \eta}{\partial \xi^{2}}+\eta^{2}(1-\eta) .
$$

In the particular case where the coefficient $C$ vanishes, Eq. (A13) has been investigated in Ref. 8. Two types of propagating front solutions are distinguished there, viz., cold and warm fronts. It is shown in Ref. 8 that the marginally stable cold fronts are characterized by

$$
\left.B=0, \quad \eta=(1 / 2)\left\{1+\tanh \left[\left(\xi-\xi_{0}\right) / 2\right)\right]\right\},
$$

while the marginally stable warm fronts are represented by

$$
\begin{gathered}
B=1, \\
\eta=\left(\xi-\xi_{0}\right)^{-1} \text { when } \xi-\xi_{0} \geqslant 1, \\
\eta=1 \text { when } \xi-\xi_{0} \leqslant 1 .
\end{gathered}
$$

The front solutions (A14) and (A15) may be extended continuously to cases where $C>0$, by means of the following expressions:

$$
\begin{gathered}
B=(1 / 4)\left[1-\left(1+8 C / A^{2}\right)^{1 / 2}\right], \\
\eta=(1 / 2)\left\{1+\tanh \left[\left(\xi-\xi_{0}\right) / 2 \lambda\right]\right\} \\
\lambda=1-2 B=-C / A^{2} B
\end{gathered}
$$

for cold fronts and

$$
\begin{gathered}
B=(1 / 2)\left[1+\left(1+2 C / A^{2}\right)^{1 / 2}\right], \\
\eta=B\left(\xi-\xi_{0}\right)^{-1} \text { when } \xi-\xi_{0} \geqslant B, \\
\eta=1 \text { when } \xi-\xi_{0} \leqslant B
\end{gathered}
$$

for warm fronts. Expressions (A16) and (A17) represent, obviously, exact solutions of Eq. (A13). An inspection of Eqs. (A16) and (A17) shows that the asymptotic behavior of the cold fronts is exponential and that of the warm fronts is algebraic. The significance of the exact solutions will be considered in more detail in a separate paper dealing with propagating fronts of superfluid turbulence.
${ }^{1}$ J.A. Geurst, Physica B 154, 327 (1989).

${ }^{2}$ J.A. Geurst and H. van Beelen, Physica A 206, 58 (1994).

${ }^{3}$ J.A. Geurst, Physica A 183, 279 (1992).

${ }^{4}$ J.A. Geurst, in Proceedings of the 1st International Workshop on Quantum Vorticity and Turbulence in He II Flows, edited by G. Stamm and W. Fiszdon (Max-Planck-Institut für Strömungsforschung, Göttingen, 1994).

${ }^{5}$ J.A. Geurst and H. van Beelen, Physica B 205, 209 (1995).

${ }^{6}$ J.T. Tough, in Progress in Low Temperature Physics, edited by D.F. Brewer (North-Holland, Amsterdam, 1982), Vol. VIII.

${ }^{7}$ K.W. Schwarz, Phys. Rev. B 31, 5782 (1985); 38, 2398 (1988).

${ }^{8}$ J.A. Geurst and H. van Beelen, Physica A 216, 407 (1995).

${ }^{9}$ R.J. Donnelly, Quantized Vortices in Helium II (Cambridge University Press, Cambridge, 1991)

${ }^{10}$ S.K. Nemirovskii and W. Fiszdon, Rev. Mod. Phys. 67, 37 (1995).

${ }^{11}$ I.M. Khalatnikov, An Introduction to the Theory of Superfluidity (Benjamin, New York, 1965).

${ }^{12}$ L.D. Landau and E.M. Lifshitz, Fluid Mechanics (Pergamon, London, 1959).
${ }^{13}$ J.D. Logan, Invariant Variational Principles (Academic, New York, 1977).

${ }^{14}$ H. Goldstein, Classical Mechanics, 2nd ed. (Addison-Wesley, Reading, 1980).

${ }^{15}$ In Eq. (7.4) of Ref. 2 the quantity $R / T$ should be replaced by $R / T-\vec{\nabla} \cdot(\vec{q} / T)$, while in Eq. (7.5) the expression $r_{\ell}-\vec{\nabla} \cdot \vec{q}_{\ell}$ has to be substituted for $r_{\ell}$.

${ }^{16}$ H. van Beelen, W. van Joolingen, and K. Yamada, Physica B 153, 248 (1988).

${ }^{17}$ S.R. de Groot and P. Mazur, Nonequilibrium Thermodynamics (North-Holland, Amsterdam, 1962).

${ }^{18}$ W.F. Vinen, Proc. R. Soc. London, Ser. A 240, 114 (1957); 240, 128 (1957); 242, 493 (1957); 243, 400 (1957).

${ }^{19}$ R.J. Donnelly, Experimental Superfluidity (The University of Chicago Press, Chicago, 1967).

${ }^{20}$ C.J. Swanson and R.J. Donnelly, J. Low Temp. Phys. 52, 189 (1983).

${ }^{21}$ We note in passing that the well-known Gorter-Mellink mutual friction force only applies when the vortex tangle is in external equilibrium.

${ }^{22}$ G.B. Whitham, Linear and Nonlinear Waves (Wiley, New York, 1974). 